\title{
The age of the oldest Open Clusters
}

\author{
M. Salaris ${ }^{1,2}$, A. Weiss ${ }^{2}$, and S. M. Percival ${ }^{1}$ \\ 1 Astrophysics Research Institute, Liverpool John Moores University, Twelve Quays House, Egerton Wharf, Birkenhead, \\ CH41 1LD, UK \\ e-mail: smp@astro.livjm.ac.uk \\ 2 Max-Planck-Institut für Astrophysik, Karl-Schwarzschild-Strasse 1, 85758, Garching, Germany \\ e-mail: weiss@mpa-garching.mpg.de
}

Received 15 August 2003 / Accepted 2 October 2003

\begin{abstract}
We determine ages of 71 old Open Clusters by a two-step method: we use main-squence fitting to 10 selected clusters, in order to obtain their distances, and derive their ages from comparison with our own isochrones used before for Globular Clusters. We then calibrate the morphological age indicator $\delta(V)$, which can be obtained for all remaining clusters, in terms of age and metallicity. Particular care is taken to ensure consistency in the whole procedure. The resulting Open Cluster ages connect well to our previous Globular Cluster results. From the Open Cluster sample, as well as from the combined sample, questions regarding the formation process of Galactic components are addressed. The age of the oldest open clusters (NGC 6791 and Be 17) is of the order of $10 \mathrm{Gyr}$. We determine a delay by $2.0 \pm 1.5 \mathrm{Gyr}$ between the start of the halo and thin disk formation, whereas thin and thick disk started to form approximately at the same time. We do not find any significant agemetallicity relationship for the open cluster sample. The cumulative age distribution of the whole open cluster sample shows a moderately significant $(\sim 2 \sigma$ level$)$ departure from the predictions for an exponentially declining dissolution rate with timescale of 2.5 Gyr. The cumulative age distribution does not show any trend with galactocentric distance, but the clusters with larger height to the Galactic plane have an excess of objects between 2-4 and $6 \mathrm{Gyr}$ with respect to their counterpart closer to the plane of the Galaxy.
\end{abstract}

Key words. Galaxy: disk - evolution - open clusters and associations: general - stellar content

\section{Introduction}

The theory of the formation of galaxies is without any doubt one of the outstanding problems of astrophysics. Although in the past decades considerable progress has been made, we do not have yet a complete and definitive picture of how galaxies form. As discussed by, e.g., Freeman \& Bland-Hawthorn (2002), a detailed study of the formation of the Galaxy lies at the core of understanding the complex processes leading to the formation of external galaxy systems. A way to shed some light on this problem is to study the timescale for the formation of the different Galactic populations, e.g., halo, thick disk, thin disk and bulge, by means of stellar age dating. The most reliable stellar ages are obtained for the star clusters belonging to the various populations, i.e., the globular clusters (GCs) in the halo, thick disk and bulge, and the open clusters (OCs) in the thin disk. The advantage of dating star clusters over individual stars - whose age determination relies entirely on the knowledge of individual metallicities, effective temperatures and gravities (or absolute magnitudes), which have to be fitted by the appropriate theoretical model - stems from the fact that star clusters are made of coeval objects, largely with the

Send offprint requests to: M. Salaris, e-mail: ms@astro.livjm.ac.uk same initial chemical composition and located at the same distance, so that it is possible to use morphological parameters deduced from theoretical isochrones in order to derive their age. In this way one can bypass the thorny problem of determining a reliable empirical and theoretical temperature scale, and of acquiring high resolution spectroscopy for large samples of stars.

In a series of papers published in the last 6 years (Salaris et al. 1997; Salaris \& Weiss 1997, 1998; Salaris \& Weiss 2002, hereinafter SW02), we have addressed the problem of the timescale for the formation of the halo and thick disk by homogeneously determining the age of a large sample of Galactic GCs. The latest SW02 study (including $55 \mathrm{GCs}$ ) concluded that metal poor clusters (up to $[\mathrm{Fe} / \mathrm{H}]$ between -1.6 and -1.2 , depending on the adopted $[\mathrm{Fe} / \mathrm{H}]$ scale) are coeval within $\sim 1 \mathrm{Gyr}$, with an age of the order of 12-13 Gyr, whereas the more metal rich ones show an age spread, are on average younger and display a weak age-metallicity relationship (age decreasing with increasing $[\mathrm{Fe} / \mathrm{H}])$. This result is in agreement with other independent analyses, such as that by Rosenberg et al. (1999). When searching for relationships between age and position within the halo, it was found that the age spread starts from galactocentric distances $\left(R_{\mathrm{gc}}\right)$ between 8 and $13 \mathrm{kpc}$ outwards, the precise value depending again on the adopted $[\mathrm{Fe} / \mathrm{H}]$ scale. 
It is now important to address the question of when the thin disk started to build up, relative to the thick disk and halo. This can be accomplished by studying the age distribution of the oldest OCs. In general, OCs are expected to be disrupted easily by encounters with massive clouds in the disk (Spitzer 1958); however, the most massive OCs or those with orbits that keep them far away from the Galactic plane for most of their lifetimes are expected to survive for longer periods of time. These old objects are therefore test particles - in analogy to the GCs - probing the earliest stages of the formation of the disk. It is essential to determine their ages using stellar models and methods which place them on the same scale as GC ages. An analysis of this kind, based on homogeneous age dating of all the known old OCs and a large sample of GCs, employing the latest generation of stellar models is still lacking (see, e.g., Liu \& Chaboyer 2000 for a study of this kind, but considering only a very small number of OCs and GCs), and this paper is intended to fill this gap.

Here we will reanalyze the old OC sample reviewed by Friel (1995), and based on the seminal papers by Phelps et al. (1994) and Janes \& Phelps (1994, hereafter JP94), to which we have added two additional clusters (ESO 093-SC08 and vdBH 176) recently studied by Phelps \& Schick (2003). This should contain approximately all presently known old OCs. Our aim is to determine their age on a scale consistent with the GC ages determined by SW02, to study the existence of possible relationships between age, position within the disk and $[\mathrm{Fe} / \mathrm{H}]$, and to compare their ages with the GC population. In Sect. 2 we describe the cluster sample and the techniques used to determine their age. The resulting age distribution is analyzed in Sect. 3, while Sect. 4 deals with the comparison with the GC ages by SW02. A summary and conclusions follow in Sect. 5 .

\section{Cluster sample and age determination method}

We consider a total of 71 clusters -69 from Friel (1995) and 2 from Phelps \& Schick (2003) - whose morphological age parameter $\delta(V)$ is equal to or larger than the value for Praesepe, i.e. $\delta(V)=0.3$ (see below for the definition of $\delta(V)$ ). The definition of old OCs by JP94 is slightly different: they considered as "old" all OCs where $\delta(V)$ is larger than zero. We have not included objects with $\delta(V)<0.3$, because we did not have clusters with $\delta(V)$ between 0 and 0.3 that could be used to calibrate adequately a relationship between this parameter and cluster age (see below for details about the calibration).

Friel's (1995) sample is mainly the same as the one studied by JP94, with only a few additions. JP94 have discussed in detail the completeness of their sample of old OCs, and concluded that most probably the number of undetected old clusters is small and should not have a major effect on the overall age distribution, even though the properties of the age distribution perpendicular to the Galactic plane may be affected by still undetected old OCs, which should be preferentially located very close to the plane of the Galaxy.

The $[\mathrm{Fe} / \mathrm{H}]$ values for our sample are taken whenever possible (38 clusters) from Gratton (2000, hereafter G00), who transformed various metallicity scales based on low

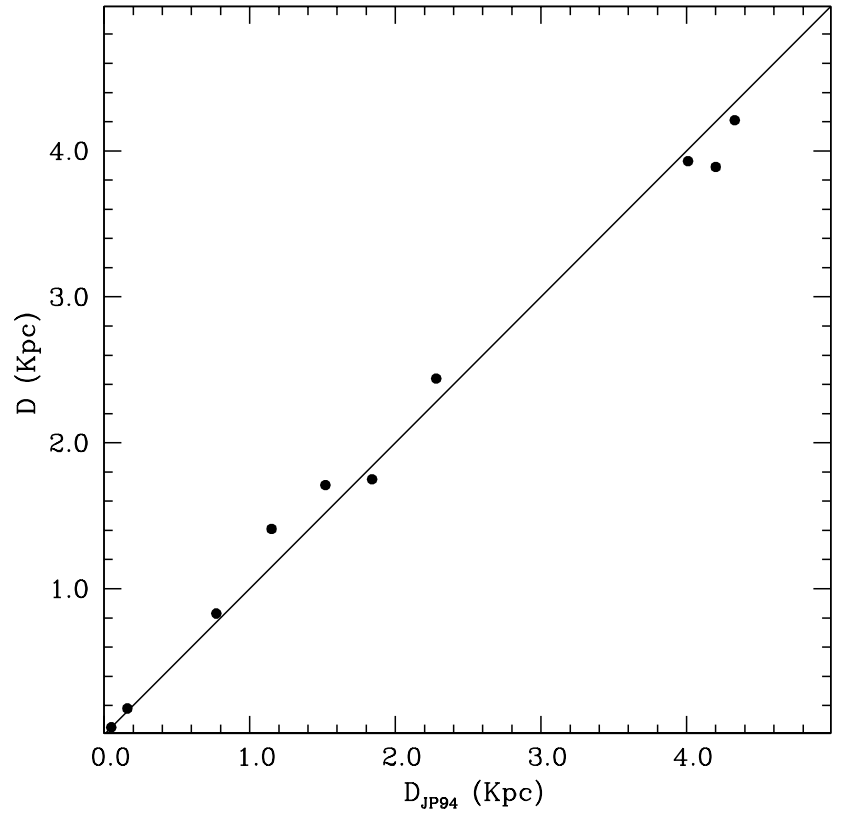

Fig. 1. Comparison between the distances given by JP94 and the MS fitting distances we obtain for a subsample of 10 clusters (see text for details). The solid line represent the 1:1 relationship between the two sets of distances.

resolution spectroscopy onto an homogeneous scale tied to high resolution $[\mathrm{Fe} / \mathrm{H}]$ determinations. One exception is the cluster Praesepe, for which we have employed the Hyades metallicity (G00 reports a lower value), based on the discussion and references in Percival et al. (2003, hereafter PSK03). In case of clusters not listed by G00 we have either used the value provided by the WEBDA OC database (http://obswww. unige.ch/webda/, see Mermilliod 1992) when available, to which we attached an error of 0.15 dex ( 11 clusters), or we assumed $[\mathrm{Fe} / \mathrm{H}]=0.0$ with an error of 0.20 dex (22 clusters). The cluster galactocentric distances and heights to the Galactic plane are taken from Friel (1995) and Phelps \& Schick (2003). For many of the clusters in our analysis the existing photometry and/or uncertainties in the cluster parameters do not allow to perform a more accurate and homogeneous distance determination. Therefore we used the results presented in the mentioned papers, where more details about this issue can be found.

As a test we compared in Fig. 1 the distances we obtained for a subsample of 10 clusters (see next subsection for details) from the Main Sequence (hereinafter MS) fitting technique, with the JP94 results. We did not find any statistically significant trend of the difference between the two sets of distances with respect to our MS fitting determinations. The mean value of the difference is equal to only $9 \mathrm{pc}$, with a dispersion of $160 \mathrm{pc}$ around the mean.

The data for the complete cluster sample are summarized in Table 1; the flag attached to each cluster provides the source for the metallicity; a value equal to 0 or 1 means $[\mathrm{Fe} / \mathrm{H}]$ from $\mathrm{G} 00$, whereas a value of 2 means that the source is the WEBDA database or that there is no available $[\mathrm{Fe} / \mathrm{H}]$ determination. A flag equal to 0 denotes the subsample of clusters that are used for our age calibration, as explained in the next subsections. 
Table 1. Cluster data. The columns display, respectively, cluster name, value of the $\delta(\mathrm{V})$ morphological parameter and its associated error, $[\mathrm{Fe} / \mathrm{H}]$ and associated error, age in $\mathrm{Gyr}$ and associated error, galactocentric distance in kpc, height to the Galactic plane in pc, source of [Fe/H] value, previous age estimate on the JP94 scale (see text for details). The last 11 clusters are the calibrating clusters for our $t$-[Fe/H]- $\delta(V)$ relationship.

\begin{tabular}{|c|c|c|c|c|c|c|c|c|c|c|}
\hline Cluster & $\delta(V)$ & $\sigma(\delta(V))$ & {$[\mathrm{Fe} / \mathrm{H}]$} & $\sigma([\mathrm{Fe} / \mathrm{H}])$ & $t$ (Gyr) & $\sigma(t)$ & $R_{\mathrm{gc}}(\mathrm{kpc})$ & $z(\mathrm{pc})$ & flag & $t_{\mathrm{JP} 94}(\mathrm{Gyr})$ \\
\hline King 2 & 2.2 & 0.15 & 0.00 & 0.20 & 5.03 & 1.31 & 12.98 & -510 & 2 & 5.6 \\
\hline IC 166 & 1 & 0.25 & -0.27 & 0.15 & 1.32 & 0.43 & 10.74 & -10 & 1 & 1.5 \\
\hline NGC 752 & 0.9 & 0.05 & -0.09 & 0.06 & 1.24 & 0.20 & 8.75 & -145 & 1 & 1.4 \\
\hline Be 66 & 2.0 & 0.25 & 0.00 & 0.20 & 3.98 & 1.52 & 12.59 & 20 & 2 & 4.4 \\
\hline NGC 1193 & 2.1 & 0.15 & -0.35 & 0.11 & 4.23 & 1.08 & 12.00 & -845 & 1 & 4.9 \\
\hline King 5 & 0.4 & 0.15 & -0.30 & 0.15 & 0.76 & 0.16 & 10.34 & -163 & 2 & 0.9 \\
\hline NGC 1245 & 0.7 & 0.15 & 0.10 & 0.15 & 1.06 & 0.23 & 11.09 & -465 & 1 & 1.0 \\
\hline NGC 1798 & 1.0 & 0.15 & -0.47 & 0.15 & 1.28 & 0.29 & 11.79 & 290 & 2 & 1.5 \\
\hline NGC 1817 & 0.8 & 0.05 & -0.10 & 0.09 & 1.12 & 0.18 & 10.26 & -410 & 1 & 1.3 \\
\hline Be 17 & 2.8 & 0.15 & -0.33 & 0.15 & 10.06 & 2.77 & 10.89 & -155 & 1 & 12.6 \\
\hline Be 18 & 2.3 & 0.15 & 0.02 & 0.15 & 5.69 & 1.49 & 12.09 & 325 & 1 & 5.6 \\
\hline Be 20 & 2.1 & 0.05 & -0.61 & 0.15 & 4.05 & 0.69 & 16.12 & -2420 & 2 & 4.9 \\
\hline Be 21 & 1.6 & 0.25 & -0.97 & 0.15 & 2.18 & 0.78 & 14.27 & -255 & 1 & 2.8 \\
\hline Be 22 & 2.1 & 0.25 & -0.30 & 0.15 & 4.26 & 1.65 & 11.92 & -530 & 1 & 3.5 \\
\hline NGC 2141 & 1.6 & 0.25 & -0.26 & 0.15 & 2.45 & 0.88 & 12.60 & -430 & 2 & 2.8 \\
\hline NGC 2158 & 1.4 & 0.15 & -0.48 & 0.11 & 1.91 & 0.45 & 12.36 & 120 & 1 & 2.2 \\
\hline NGC 2194 & 0.5 & 0.15 & 0.00 & 0.20 & 0.87 & 0.19 & 11.06 & -110 & 2 & 1.0 \\
\hline NGC 2192 & 0.6 & 0.15 & -0.31 & 0.15 & 0.91 & 0.20 & 11.88 & 635 & 2 & 1.1 \\
\hline NGC 2236 & 0.4 & 0.25 & 0.00 & 0.20 & 0.80 & 0.24 & 11.61 & -100 & 2 & 0.9 \\
\hline NGC 2243 & 2.2 & 0.15 & -0.48 & 0.06 & 4.66 & 1.20 & 10.76 & -1130 & 1 & 5.6 \\
\hline $\operatorname{Tr} 5$ & 2.3 & 0.25 & 0.00 & 0.20 & 5.67 & 2.26 & 11.13 & 50 & 2 & 4.9 \\
\hline NGC 2266 & 0.5 & 0.25 & 0.00 & 0.20 & 0.87 & 0.26 & 11.80 & 600 & 2 & 1.0 \\
\hline Be 29 & 2.1 & 0.05 & -0.18 & 0.15 & 4.34 & 0.74 & 18.72 & 1465 & 1 & 5.6 \\
\hline Be 31 & 2.3 & 0.25 & -0.40 & 0.15 & 5.32 & 2.11 & 12.02 & 340 & 2 & 3.5 \\
\hline Be 30 & 0.3 & 0.15 & 0.00 & 0.20 & 0.74 & 0.16 & 10.58 & 120 & 2 & 0.9 \\
\hline Be 32 & 2.4 & 0.15 & -0.50 & 0.15 & 5.91 & 1.56 & 11.30 & 235 & 2 & 7.2 \\
\hline To 2 & 1.5 & 0.05 & -0.45 & 0.06 & 2.13 & 0.35 & 13.08 & -725 & 1 & 2.5 \\
\hline NGC 2324 & 0.3 & 0.25 & -0.52 & 0.11 & 0.67 & 0.20 & 11.29 & 185 & 1 & 0.9 \\
\hline NGC 2354 & 0.8 & 0.25 & 0.00 & 0.20 & 1.14 & 0.36 & 9.56 & -215 & 2 & 1.3 \\
\hline NGC 2355 & 0.4 & 0.15 & -0.07 & 0.15 & 0.79 & 0.17 & 10.52 & 450 & 2 & 0.9 \\
\hline NGC 2360 & 0.5 & 0.05 & -0.14 & 0.08 & 0.85 & 0.14 & 9.28 & -30 & 1 & 1.0 \\
\hline Haf 6 & 0.3 & 0.25 & 0.00 & 0.20 & 0.73 & 0.21 & 10.91 & 15 & 2 & 0.9 \\
\hline Me 66 & 2.3 & 0.15 & -0.38 & 0.06 & 5.33 & 1.38 & 9.44 & -710 & 1 & 6.3 \\
\hline Me 71 & 0.5 & 0.15 & -0.30 & 0.06 & 0.83 & 0.18 & 10.46 & 210 & 1 & 1.0 \\
\hline AM 2 & 2.5 & 0.15 & 0.00 & 0.15 & 7.24 & 1.93 & 14.06 & -740 & 2 & 8.3 \\
\hline NGC 2506 & 1.5 & 0.05 & -0.42 & 0.09 & 2.14 & 0.35 & 10.81 & 555 & 1 & 2.5 \\
\hline Pismis 2 & 1.1 & 0.25 & -0.07 & 0.15 & 1.51 & 0.50 & 9.47 & -165 & 2 & 1.7 \\
\hline Pismis 3 & 1.7 & 0.25 & 0.00 & 0.20 & 2.84 & 1.04 & 8.83 & 10 & 2 & 3.1 \\
\hline NGC 2627 & 1.6 & 0.15 & 0.00 & 0.20 & 2.55 & 0.62 & 9.28 & 220 & 2 & 2.8 \\
\hline NGC 2660 & 0.4 & 0.15 & -0.55 & 0.11 & 0.73 & 0.16 & 9.18 & -155 & 1 & 0.9 \\
\hline NGC 2849 & 0.5 & 0.25 & 0.00 & 0.20 & 0.87 & 0.26 & 10.64 & 630 & 2 & 1.0 \\
\hline 092-SC18 & 2.2 & 0.25 & 0.00 & 0.20 & 5.03 & 1.98 & 9.00 & -740 & 2 & 5.6 \\
\hline NGC 3680 & 1 & 0.15 & 0.06 & 0.08 & 1.37 & 0.31 & 8.27 & 310 & 1 & 1.5 \\
\hline Cr 261 & 2.6 & 0.15 & -0.16 & 0.15 & 8.00 & 2.16 & 7.49 & -250 & 2 & 9.5 \\
\hline NGC 4815 & 1.1 & 0.25 & 0.00 & 0.20 & 1.52 & 0.51 & 7.90 & -80 & 2 & 1.7 \\
\hline NGC 5822 & 0.8 & 0.25 & 0.09 & 0.06 & 1.16 & 0.36 & 7.94 & 45 & 1 & 1.3 \\
\hline
\end{tabular}

We have used as age indicator the morphological parameter $\delta(V)$ defined by JP94, which is similar to the $\Delta(V)$ parameter used in GC dating (e.g. SW02), calibrated in terms of absolute age and $[\mathrm{Fe} / \mathrm{H}]$ following the same kind of approach as in JP94, and in Carraro \& Chiosi (1994a) for their analysis of a sample of 36 old OCs. 
Table 1. continued.

\begin{tabular}{|c|c|c|c|c|c|c|c|c|c|c|}
\hline Cluster & $\delta(V)$ & $\sigma(\delta(V))$ & {$[\mathrm{Fe} / \mathrm{H}]$} & $\sigma([\mathrm{Fe} / \mathrm{H}])$ & $t$ (Gyr) & $\sigma(t)$ & $R_{\mathrm{gc}}(\mathrm{kpc})$ & $z(\mathrm{pc})$ & flag & $t_{\mathrm{JP} 94}(\mathrm{Gyr})$ \\
\hline IC 4651 & 1.2 & 0.15 & 0.00 & 0.09 & 1.68 & 0.39 & 7.65 & -125 & 1 & 1.8 \\
\hline IC 4756 & 0.4 & 0.15 & -0.03 & 0.06 & 0.79 & 0.17 & 8.19 & 35 & 1 & 0.9 \\
\hline Be 42 & 0.4 & 0.25 & 0.00 & 0.20 & 0.80 & 0.24 & 7.60 & -45 & 2 & 0.9 \\
\hline NGC 6802 & 0.4 & 0.25 & 0.00 & 0.20 & 0.80 & 0.24 & 7.96 & 15 & 2 & 0.9 \\
\hline NGC 6819 & 1.7 & 0.15 & 0.15 & 0.09 & 2.91 & 0.71 & 8.18 & 300 & 1 & 3.1 \\
\hline NGC 6827 & 0.5 & 0.25 & 0.00 & 0.20 & 0.87 & 0.26 & 8.32 & -355 & 2 & 1.0 \\
\hline NGC 6939 & 1.4 & 0.25 & -0.05 & 0.11 & 2.05 & 0.71 & 8.70 & 255 & 1 & 2.2 \\
\hline Be 54 & 2.5 & 0.25 & 0.02 & 0.09 & 7.27 & 2.97 & 8.54 & -165 & 1 & 7.2 \\
\hline NGC 7044 & 0.7 & 0.15 & 0.00 & 0.20 & 1.04 & 0.23 & 9.08 & -280 & 2 & 1.2 \\
\hline Be 56 & 2.3 & 0.25 & 0.00 & 0.20 & 5.67 & 2.26 & 9.92 & -515 & 2 & 6.3 \\
\hline NGC 7142 & 2 & 0.15 & 0.09 & 0.11 & 4.04 & 1.02 & 9.70 & 485 & 1 & 4.4 \\
\hline King 9 & 2 & 0.25 & 0.00 & 0.20 & 3.98 & 1.52 & 10.41 & -145 & 2 & 4.4 \\
\hline King 11 & 2.3 & 0.15 & -0.23 & 0.15 & 5.46 & 1.43 & 9.69 & 245 & 1 & 6.3 \\
\hline 093-SC08 & 2.4 & 0.25 & 0.00 & 0.20 & 6.40 & 2.59 & 13.00 & -1000 & 2 & 7.3 \\
\hline vdBH 176 & 2.5 & 0.25 & 0.00 & 0.20 & 7.24 & 2.98 & 12.00 & 1350 & 2 & 8.6 \\
\hline \multicolumn{11}{|c|}{ Calibrating clusters } \\
\hline M 67 & 2.3 & 0.05 & 0.02 & 0.06 & 4.30 & 0.50 & 9.05 & 405 & 0 & 6.3 \\
\hline NGC 2477 & 0.5 & 0.15 & 0.00 & 0.08 & 1.00 & 0.30 & 8.89 & -115 & 0 & 1.0 \\
\hline NGC 188 & 2.4 & 0.15 & -0.03 & 0.06 & 6.30 & 0.80 & 9.35 & 580 & 0 & 7.2 \\
\hline NGC 7789 & 1.1 & 0.05 & -0.13 & 0.08 & 1.80 & 0.30 & 9.44 & -170 & 0 & 1.7 \\
\hline Be 39 & 2.4 & 0.05 & -0.15 & 0.09 & 7.00 & 1.00 & 11.71 & 700 & 0 & 7.2 \\
\hline NGC 2204 & 1.4 & 0.15 & -0.38 & 0.08 & 2.00 & 0.30 & 11.84 & -1200 & 0 & 2.2 \\
\hline NGC 2420 & 1.6 & 0.05 & -0.44 & 0.06 & 2.20 & 0.30 & 10.59 & 765 & 0 & 2.8 \\
\hline NGC 6791 & 2.6 & 0.05 & 0.40 & 0.06 & 10.20 & 1.20 & 8.12 & 800 & 0 & 9.5 \\
\hline Hyades & 0.4 & 0.05 & 0.13 & 0.06 & 0.70 & 0.10 & 8.55 & -20 & 0 & 0.9 \\
\hline Praesepe & 0.3 & 0.05 & 0.13 & 0.06 & 0.70 & 0.10 & 8.62 & 85 & 0 & 0.9 \\
\hline 47 Tuc & 2.9 & 0.05 & -0.70 & 0.10 & 10.90 & 1.40 & 7.40 & -7400 & 0 & 12.0 \\
\hline
\end{tabular}

\subsection{The morphological age index $\delta(V)$}

The use of morphological indices that quantify differences in the Colour-Magnitude Diagram (CMD) of clusters in terms of age differences is a well established technique (see, e.g., Anthony-Twarog \& Twarog 1985; JP94 for OCs; Rosenberg et al. 1999 for GCs); it allows to establish a relative age ranking among a given cluster sample, bypassing the well known difficulties with isochrone fitting methods (see, e.g., VandenBerg et al. 1990; Sarajedini \& Demarque 1990; Salaris \& Weiss 1997; SW02).

Phelps et al. (1994) and JP94 have defined two morphological parameters, called $\tilde{\delta}(V)^{1}$ and $\delta 1$ which they applied to their sample of old OCs. $\tilde{\delta}(V)$ is defined as the magnitude difference between the cluster turn-off region and the He-burning clump stars. More precisely, the reference point in the turn-off region is taken as the inflection point between the turn-off and the base of the giant branch. This point is well defined and unaffected by the presence of a binary sequence and/or field stars, according to Phelps et al. (1994). $\delta 1$ is the difference in colour index between the bluest point on the MS at the luminosity of the turn-off and the colour of the giant branch one magnitude

\footnotetext{
${ }^{1}$ In the quoted paper this quantity is denoted as $\delta(V)$.
}

brighter than the turn off luminosity. A simple linear relationship between $\tilde{\delta}(V)$ and $\delta 1$ was found by JP94 when analysing the clusters where both indices could be measured, and this was applied by the same authors to obtain an estimate for $\tilde{\delta}(V)$ for those clusters where clump stars were not identified. This estimate we will call $\delta(V)$ in the following. JP94 choose $\delta(V)$ as the primary age indicator, so that for clusters without visible clump the estimated value described above was used; for the other clusters the final $\delta(V)$ given by JP94 is the average between the observed $\tilde{\delta}(V)$ and the one computed from the $\delta 1-\delta(V)$ conversion described above.

The $\delta(V)$ data for our cluster sample are reported in Table 1; the associated errors are derived from the quality grade assigned by JP94. Following JP94 we considered errors by, respectively, $0.05 \mathrm{mag}$ for clusters graded "a", $0.15 \mathrm{mag}$ for clusters graded "b" and 0.25 mag for clusters graded "c". In case of the two clusters from Phelps \& Schick (2003) we have assumed an error by $0.25 \mathrm{mag}$.

\subsection{Calibration of $\delta(V)$}

The cluster $\delta(V)$ values given in Table 1 can be translated into absolute ages by determining a relationship $\delta(V)-t-[\mathrm{Fe} / \mathrm{H}]$, 
based on a subsample of clusters with high quality CMDs, spanning the entire $[\mathrm{Fe} / \mathrm{H}]$ and $\delta(V)$ range of the full cluster sample, and for which the age can be determined with confidence. JP94 determined a relationship between $\delta(V)$ and age neglecting the effect of metallicity - based on a sample of OCs and GCs with age determinations obtained by various authors and with a variety of methods and stellar models. In case of multiple age determinations for the same objects JP94 averaged the results from the various authors. As clearly stated by JP94, due to the heterogeneity of the calibration material, their calibration was mainly aimed at producing the ranking of the clusters in terms of relative ages.

Here we wish to obtain a new highly homogeneous and reliable calibration in terms of absolute ages, based on a consistent set of updated stellar models. We have considered a subsample of 10 OCs (clusters with the flag value equal to 0 in Table 1) plus 1 GC (47 Tuc), whose ages have been determined by fitting the CMD turn-off luminosity with theoretical isochrones, after determining their distance from an empirical MS fitting technique which employs large samples of field MS stars with accurate Hipparcos parallaxes. In this way the ages we obtain are firmly tied to the Hipparcos distance scale.

The stellar models used to determine the OC ages have been computed with exactly the same updated physics employed for calculating the GC isochrones by Salaris \& Weiss (1998); these GC isochrones have been used to determine the ages of the large GC sample analyzed by SW02, and we refer the reader to Salaris \& Weiss (1998) for details about the model input physics. The turn-off stars in the younger clusters in our sample do have convective cores, and therefore we have included in our models overshooting beyond the formal boundary of the convective core (i.e., instantaneous mixing and radiative temperature gradient in the overshooting region beyond the boundary of the convective core fixed by the Schwarzschild criterion), with an extension of 0.2 pressure scale heights for masses above $1.4 M_{\odot}$, and linearly decreasing to zero from $M=1.4 M_{\odot}$ to $M=1.0 M_{\odot}$. This prescription is in broad agreement with the results obtained by Ribas et al. (2000) from the comparison of stellar models with eclipsing binary systems, at least in the mass interval spanned by the stars evolving in the turn-off region of our OC sample. We have computed stellar models and isochrones for the appropriate metallicity (scaled solar Grevesse \& Noels 1993 heavy element distribution) of each of our calibrating clusters, using an He-mass fraction $Y$ that follows the relationship $Y=0.248+1.44 Z$. The primordial $\mathrm{He}$ is derived from the recent results of analyses of the CMB power spectrum (see, e.g., the discussion in Cassisi et al. 2003), whereas the slope $\Delta(Y) / \Delta(Z)=1.44$ arises from the constraint imposed by the initial He-abundance of the standard solar model. In case of 47 Tuc and the other GC ages discussed in Sect. 4 we have computed selected $\alpha$-enhanced isochrones (the same metal distribution as in Salaris \& Weiss $1998)$ at various metallicities and with the same $Y-Z$ relationship as for the OCs, in order to determine the age of 47 Tuc and revise the ages of the GCs in SW02 which were computed using $Y=0.230+3.0 Z$.

The MS fitting distances to the calibrating OCs are from PSK03 and Percival \& Salaris (2003, hereafter PS03),
Table 2. Distance moduli and reddenings of the calibrating clusters (see text for details).

\begin{tabular}{lcr}
\hline \hline Cluster & $E(B-V)$ & $(m-M)_{0}$ \\
\hline M 67 & 0.04 & $9.60 \pm 0.09$ \\
NGC 2477 & 0.23 & $10.74 \pm 0.08$ \\
NGC 188 & 0.09 & $11.17 \pm 0.08$ \\
NGC 7789 & 0.29 & $11.22 \pm 0.07$ \\
Be 39 & 0.11 & $12.97 \pm 0.09$ \\
NGC 2204 & 0.08 & $13.12 \pm 0.08$ \\
NGC 2420 & 0.05 & $11.94 \pm 0.07$ \\
NGC 6791 & 0.15 & $12.96 \pm 0.10$ \\
Hyades & 0.00 & $3.33 \pm 0.05$ \\
Praesepe & 0.02 & $6.32 \pm 0.05$ \\
47 Tuc & 0.04 & $13.25 \pm 0.07$ \\
\hline
\end{tabular}

with the exception of NGC 6791 (see below), whilst the distance to 47 Tuc is from Percival et al. (2002, hereafter P02); they are all based on two large samples of unevolved field MS stars with accurate Hipparcos parallaxes and individual metallicity determinations. The more metal rich sample of 54 dwarfs with $[\mathrm{Fe} / \mathrm{H}]$ between $\sim-0.4$ and $\sim+0.3$ (field dwarfs) has been used to derive the distances to the calibrating OCs, while the distance to 47 Tuc has been obtained using a sample of 43 more metal-poor dwarfs with $[\mathrm{Fe} / \mathrm{H}]$ between $\sim-1.0$ and $\sim-0.3$ (sub$d$ warfs). Table 2 contains the results from the MS fitting distance determinations; reddenings are from Twarog et al. (1997) with an associated error of \pm 0.02 mag as adopted by Sarajedini (1999). It is important to notice that, as discussed in PSK03, our MS fitting distances to the Hyades and Praesepe agree well with the Hipparcos parallax measurements.

A detailed description of the distance determination method is given in the three papers mentioned above, together with the sources for the adopted MS CMDs. We just recall here that the method is based on constructing an empirical template MS from the field stars, by applying colour shifts to the individual objects to account for the differences in metallicity between the field stars and the cluster. The template is then shifted in magnitude to match the dereddened and extinction-corrected cluster MS, the extent of the shift being equal to the distance modulus $(m-M)_{0}$. The colour shifts for the field star sample have been derived empirically as discussed in PSK03 and, strictly speaking, they are applicable only within the metallicity range spanned by the field dwarfs themselves, i.e., up to $[\mathrm{Fe} / \mathrm{H}] \sim 0.3$. In case of the subdwarf sample used for 47 Tuc, the shifts have been obtained from the differential use of the isochrones by Salaris \& Weiss (1998) as discussed in P02. Whenever possible we have derived MS fitting distances using both the $(B-V)$ and $(V-I)$ colours, always finding agreement (within the associated error bars, which, for given reddening and $[\mathrm{Fe} / \mathrm{H}]$ values, are typically of a few hundredths of magnitude, and usually the same for both colours) between the two values. In this case the final distance is the unweighted mean between the results in $(B-V)$ and in $(V-I)$. The final error 
budget takes into account the errors in the cluster reddening and $[\mathrm{Fe} / \mathrm{H}]$ quoted in Tables 1 and 2 .

P02 and PSK03 discussed at length the consistency between the metallicity scale of the field stars and clusters for each of the two separate samples, a necessary prerequisite for the reliability of the MS fitting distances. What matters most in our case is the consistency between the distances obtained separately for 47 Tuc with the subdwarf sample, and for the other calibrating OCs with the field star sample. In fact, they have been determined from two different samples of field MS stars, with metallicity scales determined independently, and using different methods to determine the colour shifts. Consistency between the two sets of distances means that one should be able to use the subdwarf sample to derive the MS fitting distance modulus to a cluster like, e.g., NGC 2420, which is at the lower metallicity end of the calibrating OCs, and recover the value obtained employing the field dwarf sample $(11.94 \pm 0.07 \mathrm{mag})$. Since we get consistent distances in both $(B-V)$ and $(V-I)$ for the distance to 47 Tuc and to other calibrating OCs, we performed this test with the $(V-I)$ colour only. If consistency is achieved for this colour, it is automatically ensured for $(B-V)$, too.

We performed this test using the entire subdwarf sample and determining the appropriate colour shifts from the theoretical isochrones discussed before. We finally obtained a distance modulus that is within $0.02 \mathrm{mag}$ of the value obtained from the field dwarf sample, confirming the consistency between the distances obtained from the two separate samples of subdwarfs and field dwarfs.

We also included NGC 6791 in the calibrating sample, in order to extend our calibration to very high metallicities, and have another extremely "old" (e.g. JP94) calibrating cluster in addition to 47 Tuc. We used $E(B-V)=0.15 \pm 0.02$ (Twarog et al. 1997), $[\mathrm{Fe} / \mathrm{H}]=0.40 \pm 0.06(\mathrm{G} 00)$, and employed the same field dwarf sample and method as for the other OCs; the cluster MS CMD is from the new photometry by Stetson et al. (2003). We determined the distance modulus using both the $(B-V)$ and $(V-I)$ colours (they provide the same distance modulus within $0.01 \mathrm{mag})$, obtaining $(m-M)_{0}=12.96 \pm 0.10$, as reported in Table 2.

As a note of caution we notice that the cluster NGC 6791 metallicity is slightly above the upper limit of the $[\mathrm{Fe} / \mathrm{H}]$ range where our MS fitting method is applicable, so that we had to slightly extrapolate the empirical colour shifts applied to the field dwarfs. However, we have tested that the exclusion of NGC 6791 from the calibrating sample does not alter substantially the calibration of the sought $\delta(V)-t-[\mathrm{Fe} / \mathrm{H}]$ relationship and therefore we retained NGC 6791 in our calibrating sample.

After the MS fitting distances have been determined, cluster ages for the calibrating clusters have been obtained from isochrone fitting to the CMD turn off region, and are given in Table 1. Figure 2 shows an example (the cluster NGC 2420) of our age determination. The error bar for the age includes in quadrature the contributions due to the uncertainty in the cluster distance modulus and metallicity.

We notice that our ages are very similar to the values obtained by PS03 using the same distance moduli but the isochrones by Girardi et al. (2000). We also, as a test,

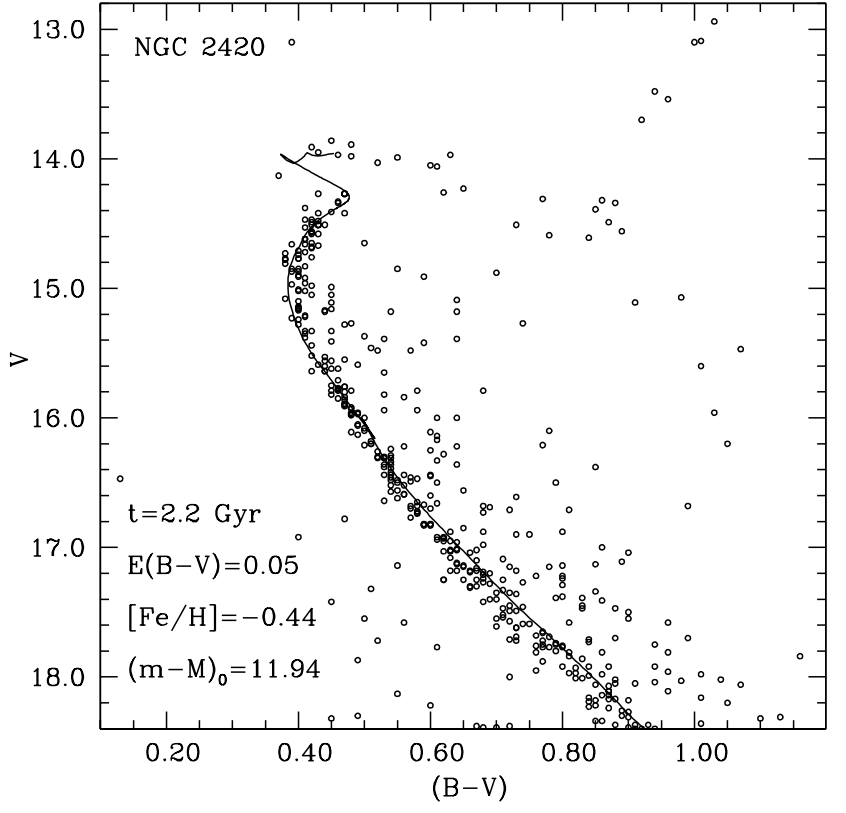

Fig. 2. Best-fitting isochrone for the MS and turn-off region of the cluster NGC 2420. Reddening, distance modulus, age and metallicity employed in the fit are given.

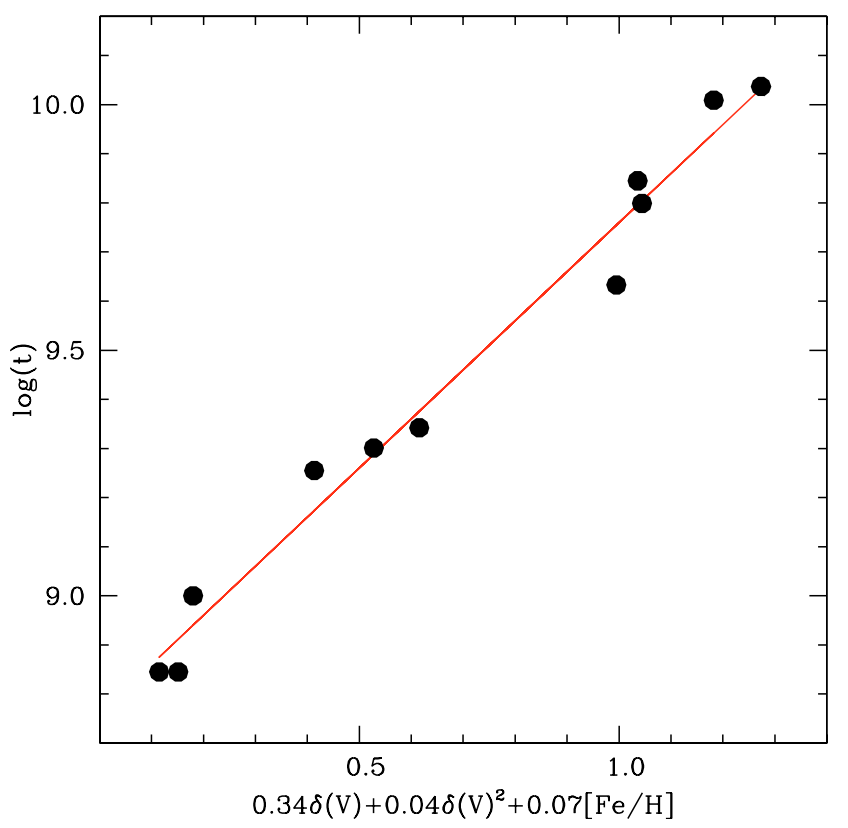

Fig. 3. Fit to the ages of the calibrating clusters (filled circles) given in Table 1.

determined the ages of our calibrating clusters by means of the Lejeune \& Schaerer (2001) isochrones, obtaining ages within less than $10 \%$ of the values given in Table 1 .

With ages, $[\mathrm{Fe} / \mathrm{H}]$ (all on the G00 scale) and $\delta(V)$ values of the calibrating clusters we determined the sought calibration of age as a function of $\delta(V)$ and metallicity. We found a simple relationship (shown in Fig. 3) between the logarithm of the cluster age and both $[\mathrm{Fe} / \mathrm{H}]$ and $\delta(V)$, given by

$\log (t)=0.04 \delta(V)^{2}+0.34 \delta(V)+0.07[\mathrm{Fe} / \mathrm{H}]+8.76$

with a $1 \sigma$ dispersion equal to 0.062 dex. 
The dependence of the logarithm of the age on $\delta(V)$ is not very different from the calibration by JP94, who found $\log (t)$ to be proportional to $0.256 \delta(V)+0.0662 \delta(V)^{2}$, without including a metallicity term. Interestingly, Carraro \& Chiosi (1994a) calibrated $\log (t)$ in terms of a morphological parameter similar to $\delta(V)$, and determined a dependence on metallicity equal to $0.08[\mathrm{Fe} / \mathrm{H}]$, almost identical to our result for the JP94 $\delta(V)$.

It is interesting to notice that the $[\mathrm{Fe} / \mathrm{H}]$ dependence of Eq. (1) is qualitatively the same as in theoretical models. In fact, by using, e.g., the Girardi et al. (2000) isochrones, we have computed the magnitude difference between He-burning clump and turn off for the age and $[\mathrm{Fe} / \mathrm{H}]$ range of the studied clusters. We found that, for a given value of this magnitude difference, metal poorer isochrones provide lower ages, as predicted by Eq. (1). The reason is that in this $[\mathrm{Fe} / \mathrm{H}]$ range, once the age is fixed, a decrease of the metallicity increases the clump luminosity more than the turn off one. Owing to the fact that the clump level is weakly dependent on age for our relevant age range, it is therefore clear that isochrones with lower metallicity have to be younger in order to show the same clump-turn off magnitude difference as more metal rich ones. The opposite happens in the regime of metal poor globular clusters, where the dependence of the horizontal branch magnitude on metallicity is weaker than the turn off one (at fixed age).

\section{The age of the old OCs}

In order to determine the ages of the remaining $61 \mathrm{OCs}$ in our sample we have applied Eq. (1) to their $\delta(V)$ and $[\mathrm{Fe} / \mathrm{H}]$ values displayed in Table 1.

The error bar on the individual determination has been computed by combining in quadrature the contribution to the uncertainty arising from the dispersion associated to Eq. (1), plus the contribution due to the $[\mathrm{Fe} / \mathrm{H}]$ and $\delta(V)$ error propagation through Eq. (1). The uncertainty in the metallicities does not play a significant role in the error budget, due to the weak dependence of $\log (t)$ on $[\mathrm{Fe} / \mathrm{H}]$.

In Fig. 4 we plot the cluster ages against their $[\mathrm{Fe} / \mathrm{H}]$. Open squares denote the 11 calibrating clusters ( 10 OCs plus 47 Tuc) which cover the entire $[\mathrm{Fe} / \mathrm{H}]$ and age range spanned by the full sample, with the only exception of Be 21 , which has a metallicity $[\mathrm{Fe} / \mathrm{H}] \sim-1.00$, and is therefore outside the range of validity of Eq. (1); thus its age has to be treated with caution.

Be 17 and NGC 6791 appear to be the oldest known OCs, and their ages are formally the same, within the error bars, as the age of the thick disk GC 47 Tuc (see Sect. 4 for a discussion about the comparison with GC ages). Figure 5 compares our ages with the results by JP94. Our values are, with few exceptions, systematically lower, with the oldest clusters Be 17 and NGC 6791 - having ages of about $10 \mathrm{Gyr}$, whereas the oldest cluster in JP94 is Be 17, with an estimated age of 12.6 Gyr, about 2.5 Gyr higher than our results; our age for Be $17(10.1 \pm 2.8 \mathrm{Gyr})$ is in line with the recent analysis by Carraro et al. (1999) who determined a value of $9 \pm 1 \mathrm{Gyr}$ from isochrone fitting, although our estimate (based on the $\delta(V)$ provided by JP94) has a much larger error bar. Our age for NGC $6791-10.2 \pm 1.2 \mathrm{Gyr}-$ is larger than the result by Chaboyer et al. (1999) who found 8.0 $\pm 0.5 \mathrm{Gyr}$ from isochrone

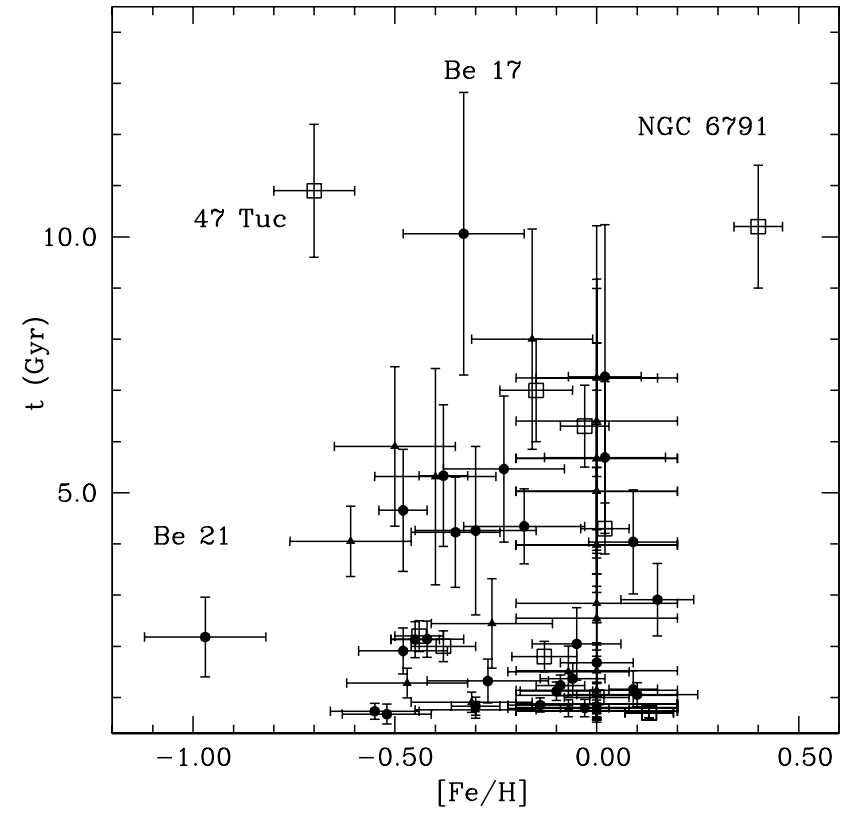

Fig. 4. Ages for the 71 old OCs plus 47 Tuc, as a function of the cluster $[\mathrm{Fe} / \mathrm{H}]$. Open squares denote the 11 calibrating clusters. Individual clusters discussed in the text are labelled.

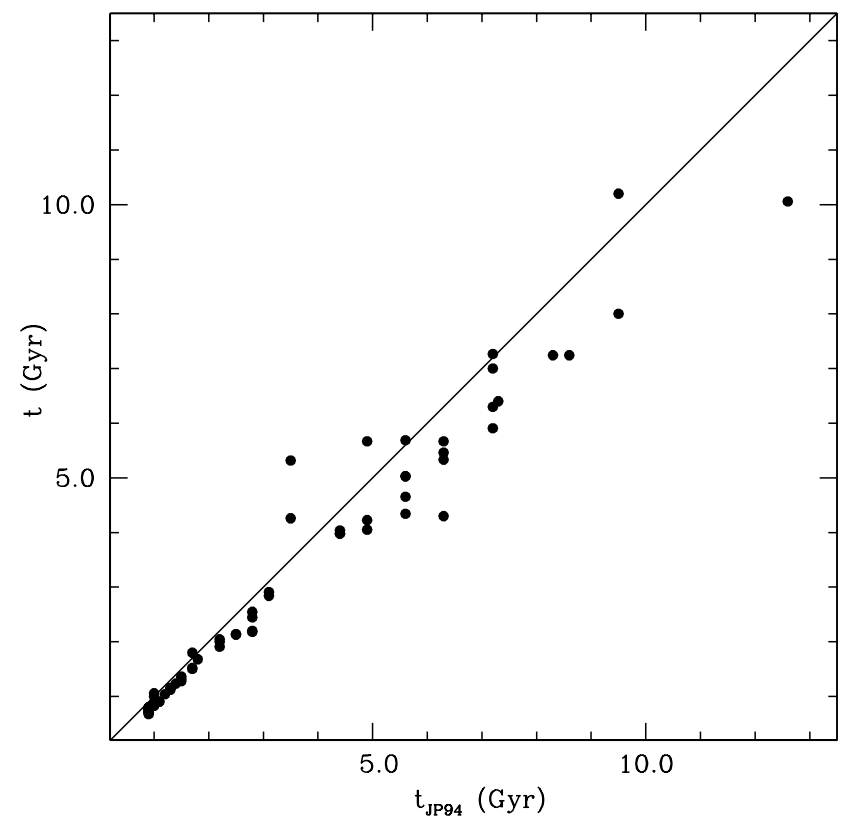

Fig. 5. Comparison between our derived ages and those given by JP94. The solid line denotes the 1:1 relationship.

fitting, and compatible, within the error bar, with the age of 8-9 Gyr determined by Carraro et al. (1999).

The lower panel of Fig. 6 shows a histogram of the cluster ages (without the GC 47 Tuc, that in the following will always be excluded from the analysis of the OC sample). As mentioned before, according to JP94 the shape of this distribution for the whole sample should not be altered by the still undetected old OCs. The corresponding observed cumulative function (i.e., number of clusters with ages larger than a given value $t_{i}$ ) is displayed in the upper panel of the same figure (open squares). 


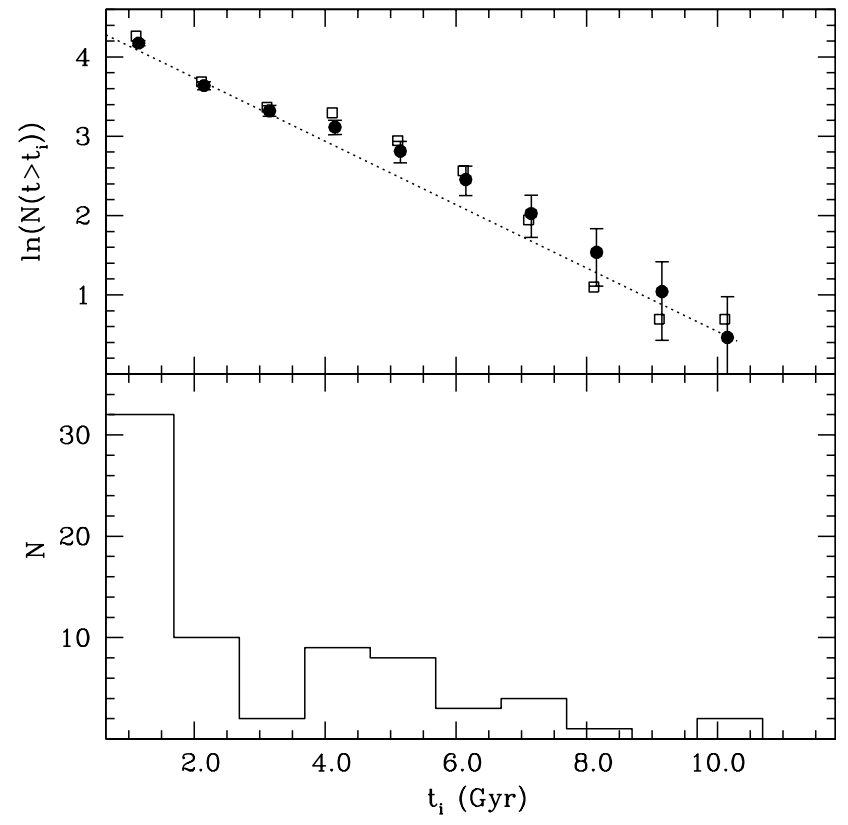

Fig. 6. Cumulative age distribution (upper panel) and differential age distribution (lower panel) for the 71 OCs in our sample. In the upper panel the age distribution from the Monte Carlo simulations discussed in the text (filled circles) is shown together with the actual cumulative distribution (open squares). The line with slope corresponding to a dissolution timescale of $2.5 \mathrm{Gyr}$ is also displayed.

These data contain in principle important information about the timescales of cluster destruction. In the simplest case of a uniform formation rate and exponentially declining dissolution rate, the open squares in the upper panel of Fig. 6 should follow a straight line whose slope is equal to the inverse of the dissolution timescale (see, e.g., Janes et al. 1988). In fact, the situation may be more complex, for the points in the cumulative age distribution apparently do not follow a single slope; there is a change in the shape of the cumulative age distribution, visible between $\sim 4$ and $\sim 6$ Gyr, which appears as a "bump" in the differential age distribution. This "excess" of clusters has been already noticed by JP94, and it was located in the age interval between 5 and 7 Gyr on their age scale. We have investigated further this matter by evaluating the error bars associated to the points in our cumulative age distribution. For this purpose, we performed an extensive Monte Carlo simulation by considering the individual OC ages given in Table 1, together with their associated errors. We then determined 10000 synthetic samples of ages for our 71 clusters. In each sample the individual cluster ages were randomly assigned according to a Gaussian distribution centred around the values given in Table 1, with a $1 \sigma$ dispersion equal to the estimated individual errors. We then determined the cumulative age distribution for each of the 10000 synthetic samples - using the same age bins as in Fig. 6-and determined the mean number counts and associated $1 \sigma$ dispersion in each age bin (an analogous result is obtained if we use the modal value of the number counts in each bin).

The upper panel of Fig. 6 displays the resulting synthetic cumulative age distribution as filled circles. It is worth noticing that in general the actual distribution lies comfortably within

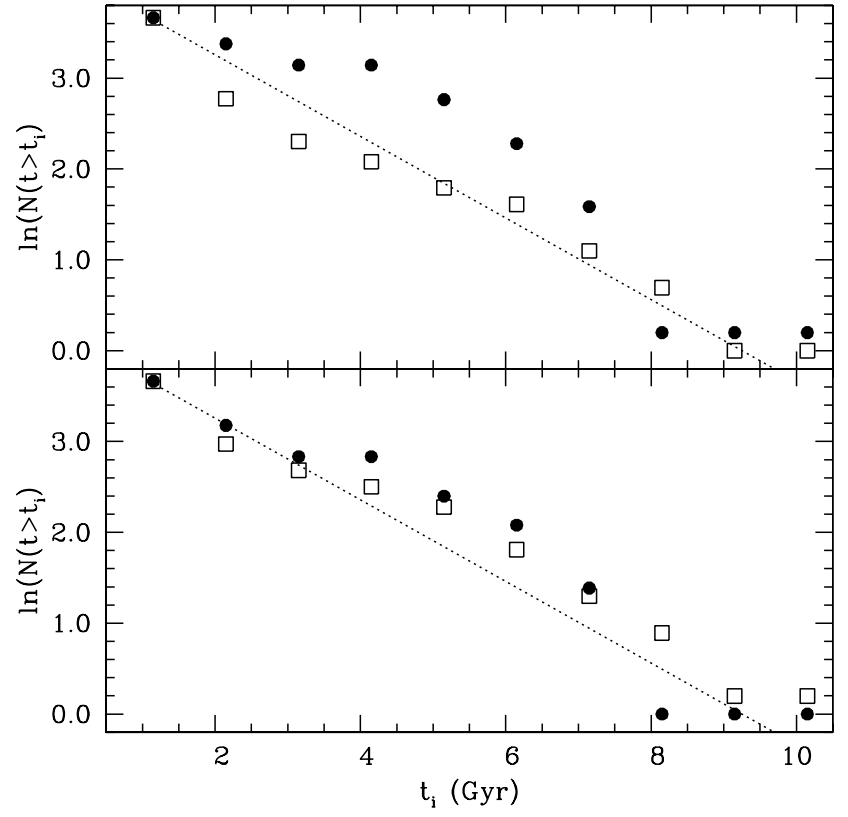

Fig. 7. Comparison of the cumulative age distributions for clusters located in two different ranges of height above the Galactic plane, $|z|$ (upper panel), or galactocentric distance, $R_{\mathrm{gc}}$ (lower panel; see text for details). The line with a slope corresponding to a dissolution timescale of $2.5 \mathrm{Gyr}$ is displayed in both panels.

the $1 \sigma$ error bars associated to the synthetic one, as expected, since it has to correspond to one realization of our ensemble of synthetic age distributions. We have also displayed the best fit single slope that matches the data: it corresponds to a dissolution timescale of $2.5 \mathrm{Gyr}$. Once the effect of the age errors is taken into account, we used a $\chi^{2}$ test to determine the significance of the excess of clusters in the age range between 4 and 6 Gyr, which results to be at $\sim 2 \sigma$ level for the whole sample of objects.

We have also studied the dependence of the cumulative age distribution on the cluster spatial positions. The lower panel of Fig. 7 shows the case for the clusters located at $R_{\mathrm{gc}}>10 \mathrm{kpc}$ (filled circles -32 objects) and at $R_{\mathrm{gc}} \leq 10 \mathrm{kpc}$ (open squares 39 objects), respectively. The age distribution for clusters with $R_{\mathrm{gc}}>10 \mathrm{kpc}$ has been rescaled to have the same total number of objects as for $R_{\mathrm{gc}} \leq 10 \mathrm{kpc}$. The two distributions of points are very similar (the result is independent of the definition of the $R_{\mathrm{gc}}$ ranges). As a guideline, the best fit single slope corresponding to a dissolution timescale of $2.5 \mathrm{Gyr}$ is also displayed. This similarity results from the lack of correlation between age distribution and $R_{\mathrm{gc}}$ for the full sample; there is no trend of age with respect to $R_{\mathrm{gc}}$, with a large age spread at any value of the galactocentric distance. All of this, in turn, suggests that the cluster formation and destruction processes are apparently not correlated with the galactocentric distance.

The upper panel of Fig. 7 shows the cumulative age distribution for clusters in two selected ranges of height above the Galactic plane $|z|$. Open squares are clusters with $|z|$ equal to or lower than $300 \mathrm{pc}$ (39 objects), whilst filled circles represent clusters at higher distances from the plane of the Galaxy (32 objects). Again, the two age distributions have been rescaled to 
the same number of objects. It is evident that in this case the two cumulative functions are different, i.e. the clusters closer to the plane follow a relationship much closer to the linear slope, corresponding to a dissolution timescale of $2.5 \mathrm{Gyr}$. The more distant ones show a clear excess of clusters in the range between 2-4 and 6 Gyr. This is different from the conclusion we drew from the distribution for the whole sample (Fig. 6), the inconclusiveness found there might come from mixing two different subsamples. Analogous results are found when changing the limits to $250 \mathrm{pc}$ or $350 \mathrm{pc}$. The difference is significant (at more than $3 \sigma$ level), even considering the error bars we obtain with a Monte Carlo simulation similar to the one discussed for the whole sample. This result hints at a more homogeneous creation-destruction processe for the clusters closer to the Galactic plane, than for their more distant counterparts. However, one has to take into account the possibility that this difference is, at least partially, an artifact due to the possible incompleteness of the OC sample at low $|z|$ (as discussed in JP94), and/or to the cluster orbital motions. The analysis by Carraro \& Chiosi (1994b) of the orbits of 5 old OCs seem to indicate that the observed $|z|$ for the old OCs do not reflect their initial values, due to the rapid oscillatory motions of the clusters across the disk.

\subsection{Age- $[\mathrm{Fe} / \mathrm{H}]$ correlation}

The determination of the age-metallicity relationship for halo and disk objects has been the subject of numerous studies, because it poses a constraint to the chemical evolution history of the Galaxy (e.g., Twarog 1980; Edvardsson et al. 1993; Carraro \& Chiosi 1994; Friel 1995, and references therein). A first glance at Fig. 4 does not show any trend of the cluster age with respect to $[\mathrm{Fe} / \mathrm{H}]$. A more detailed analysis, however, needs to take into account the radial abundance gradient present in the Galactic disk (e.g. Friel 1995, and references therein). Therefore, we have first determined the relationship - if any between cluster metallicity and galactocentric distance; we restricted our analysis to the sample of 38 clusters with $[\mathrm{Fe} / \mathrm{H}]$ on the homogeneous scale by G00 - displayed in Fig. 8 - and we fitted to the data a linear relationship weighting the various points according to the individual $[\mathrm{Fe} / \mathrm{H}]$ error, thus obtaining

$[\mathrm{Fe} / \mathrm{H}]=(-0.055 \pm 0.019) R_{\mathrm{gc}}+(0.37 \pm 0.20)$

with a statistically significant slope, in very good agreement with the value $-0.059 \pm 0.010 \mathrm{dex} \mathrm{kpc}^{-1}$ estimated by Friel et al. (2002) in their sample of 39 clusters.

The data displayed in Fig. 8 also clearly show that the value of the slope might be affected by the most distant cluster in the sample, i.e. Be 29. If we exclude this cluster the slope becomes steeper

$[\mathrm{Fe} / \mathrm{H}]=(-0.097 \pm 0.023) R_{\mathrm{gc}}+(0.77 \pm 0.23)$

but still within the range of independent determinations (see, e.g., the discussion by Friel et al. 2002).

We also tried to assess a possible dependence of this slope on the cluster ages, as found by Friel et al. (2002). As a first test, we divided the sample into two age ranges, i.e. clusters with

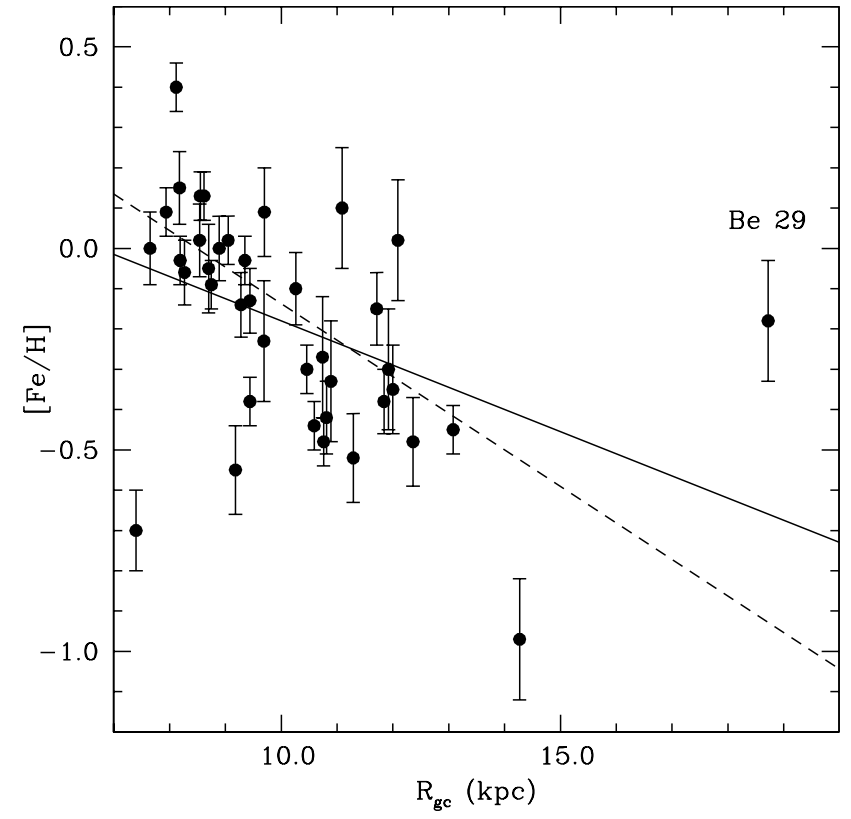

Fig. 8. Relationship between $[\mathrm{Fe} / \mathrm{H}]$ and galactocentric distance for the 38 clusters with metallicities on the G00 scale. The solid line displays the linear best-fit considering all 38 clusters; the dashed line shows the best-fit excluding Be 29 .

$t \leq 3 \mathrm{Gyr}$ and clusters older than $3 \mathrm{Gyr}$. For the first group we found a gradient $\Delta[\mathrm{Fe} / \mathrm{H}] / \Delta R_{\mathrm{gc}}=-0.13 \pm 0.02 \mathrm{dex} \mathrm{kpc}^{-1}$, and for the second one $\Delta[\mathrm{Fe} / \mathrm{H}] / \Delta R_{\mathrm{gc}}=-0.08 \pm 0.03 \mathrm{dex} \mathrm{kpc}^{-1}$. These two values are different at about $1.5 \sigma$, but the higher age group shows a flatter gradient. This is the opposite as found by Friel et al. (2002) who determined a steeper gradient for clusters older than $3 \mathrm{Gyr}$, with respect to younger objects. This could be due to the different $[\mathrm{Fe} / \mathrm{H}]$ scale they used, although their different cluster ages may also play a role.

The precise value of the gradient for the older group is again affected by Be 29; if we neglect this cluster the gradient becomes $-0.024 \pm 0.052 \mathrm{dex} \mathrm{kpc}^{-1}$.

This analysis clearly underscores the need to increase future sampling of clusters with $[\mathrm{Fe} / \mathrm{H}]$ on an homogeneous scale, in order to conclusively determine the dependence of the $[\mathrm{Fe} / \mathrm{H}]$ radial gradient on age. On the other hand, models of Galactic chemical evolution do not provide a conclusive prediction about the age dependence of the radial $[\mathrm{Fe} / \mathrm{H}]$ gradient. As shown by Tosi (1996), various authors predict a $[\mathrm{Fe} / \mathrm{H}] \mathrm{ra}-$ dial gradient which can stay constant with time, increase or decrease (e.g. Fig. 5 in Tosi 1996).

We also checked the possible existence of an $[\mathrm{Fe} / \mathrm{H}]$ gradient with respect to the height to the Galactic plane; we considered the $[\mathrm{Fe} / \mathrm{H}]$ values the clusters would display at the solar galactocentric distance (assumed to be equal to $8.5 \mathrm{kpc}$ ), by applying the radial gradient correction $\Delta[\mathrm{Fe} / \mathrm{H}] / \Delta R_{\mathrm{gc}}=$

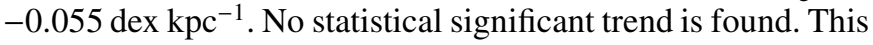
result is the same as found by Carraro \& Chiosi (1994a) in a smaller sample of old OCs; according to Carraro \& Chiosi (1994b), this may be explained in terms of rapid oscillatory motions of the clusters across the Galactic plane, which tend to erase any preexisting gradient. On the other hand, the same 


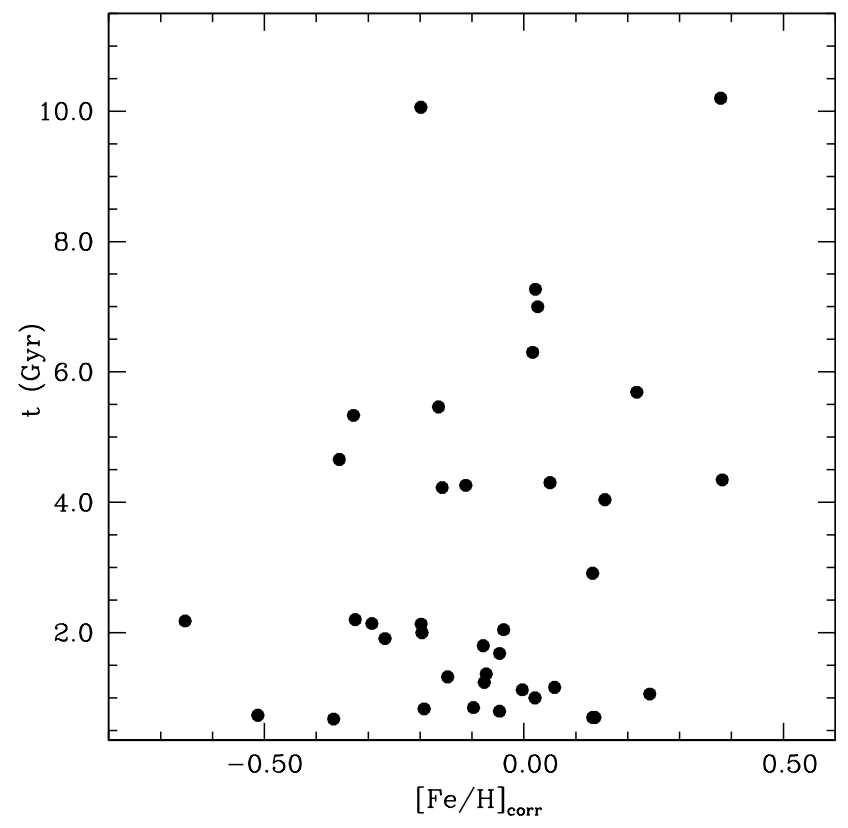

Fig. 9. Age distribution as a function of $[\mathrm{Fe} / \mathrm{H}]_{\text {corr }}([\mathrm{Fe} / \mathrm{H}]$ corrected to the solar circle, see text for details) for the 38 clusters with $[\mathrm{Fe} / \mathrm{H}]$ on the G00 scale.

authors conclude that the observed radial abundance gradients should not be seriously affected by the orbital motions, at least in the limit of the small sample of cluster orbits (5 clusters) they analyzed.

Based on these results, we have again considered the individual $[\mathrm{Fe} / \mathrm{H}]$ values of our $38 \mathrm{OCs}$ with $\mathrm{G} 00$ estimates, corrected to the solar circle $\left([\mathrm{Fe} / \mathrm{H}]_{\text {corr }}\right)$. The relationship between cluster age and $[\mathrm{Fe} / \mathrm{H}]_{\text {corr }}$ is displayed in Fig. 9, and it does not show any statistically significant trend between these two quantities, just a large age spread at any metallicity. This is consistent with the OC results by JP94, Carraro \& Chiosi (1994a), Friel et al. (2002), and also with the findings by Edvardsson et al. (1993) in a sample of field disk stars (although other studies find a significant age-metallicity relationship in field disk stars, e.g. Twarog 1980).

\section{Comparison between GC and old OC ages}

A comparison between the ages of the oldest OCs and the GCs in the thick disk and halo provides vital clues to the scenario for Galaxy formation. For this comparison to be meaningful it is however necessary to ensure that the $\mathrm{OC}$ and GC ages are on a consistent scale.

In SW02 we have accurately and homogeneously determined the ages of a large sample of 55 GCs, using stellar models computed with the same code and the same input physics as the models used in this paper. The differences with respect to this work are the age dating method and the different He enrichment law assumed in the model computation, as discussed previously. This latter point has been addressed by redetermining the age of the SW02 sample using models computed with the same He enrichment law used for the OCs. The net effect is to cause an average decrease by $0.7 \mathrm{Gyr}$ of the GC ages with respect to SW02 results.

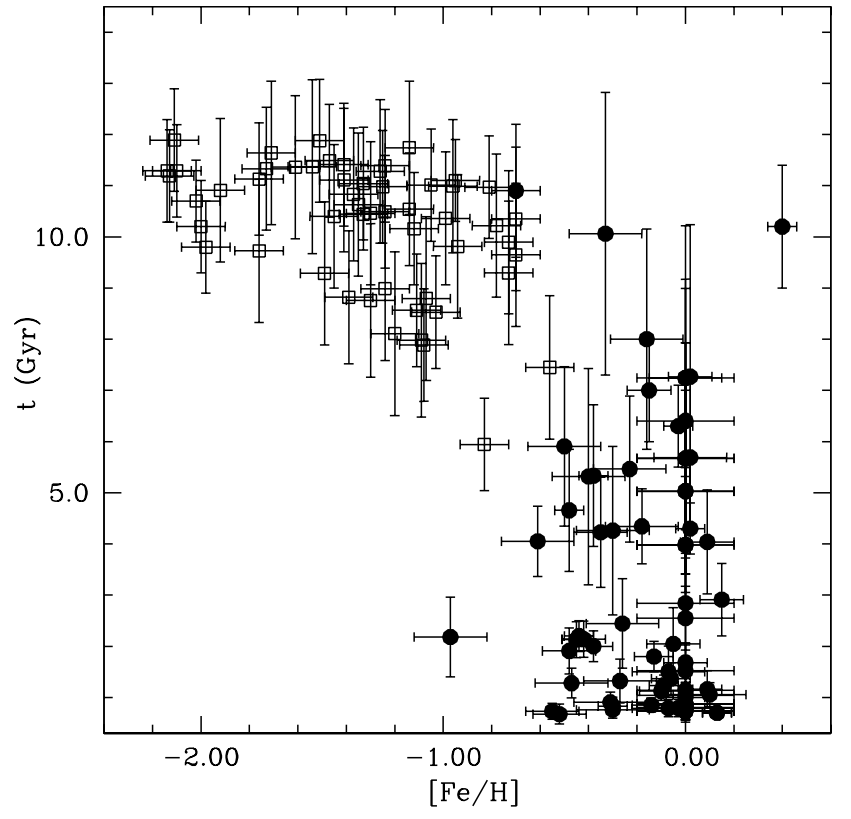

Fig. 10. Age distribution as a function of $[\mathrm{Fe} / \mathrm{H}]$ for the clusters analyzed in this study (filled circles) and the GCs studied by SW02 (open squares).

As for the different age dating methods applied to the two samples, we have one cluster - 47 Tuc - in common, whose age has been derived with both techniques. A comparison of its age given in Table 1 with the values from SW02 corrected for the new He abundances, provides a difference of only $0.5 \mathrm{Gyr}$ (SW02 age being lower), well within the error bars associated to the individual determinations.

Figure 10 shows the distribution of ages as a function of the observed $[\mathrm{Fe} / \mathrm{H}]$ for the SW02 sample (with metallicities according to the Carretta \& Gratton 1997 scale) and the old OCs of Table 1, the corresponding number counting as a function of age are displayed in Fig. 11. The cut-off in the age distribution of GCs at $\sim 12 \mathrm{Gyr}$ and the overlap between the tails of the distribution of GC and OC ages is evident. The youngest GCs, supposed to have been accreted by our Galaxy, have ages comparable to the ages of the old OCs. This means that these accretion processes were acting well after the formation of the Galactic disk. NGC 6791 and Be 17, the two oldest OCs, have formally the same age as thick disk GCs like 47 Tuc and M 71, implying an approximately coeval formation for both thin and thick disk. By comparing the ages of the oldest OCs with the oldest GCs one derives a difference between the start of the formation of the halo and of the thin disk of $\sim 2 \mathrm{Gyr}$.

\section{Summary and conclusions}

In this paper we have extended our previous age determinations for GCs to old OCs belonging to the Galactic thin disk, using as before a morphological age indicator. In case of the old OCs, it is the so-called $\delta(V)$ parameter defined by JP94. We derived a new and homogeneous calibration of the $\delta(V)-t-[\mathrm{Fe} / \mathrm{H}]$ relationship from a subsample of 10 clusters with accurate and deep photometry, $[\mathrm{Fe} / \mathrm{H}]$ and reddening estimates. Distances to these calibrating clusters have been determined by means 


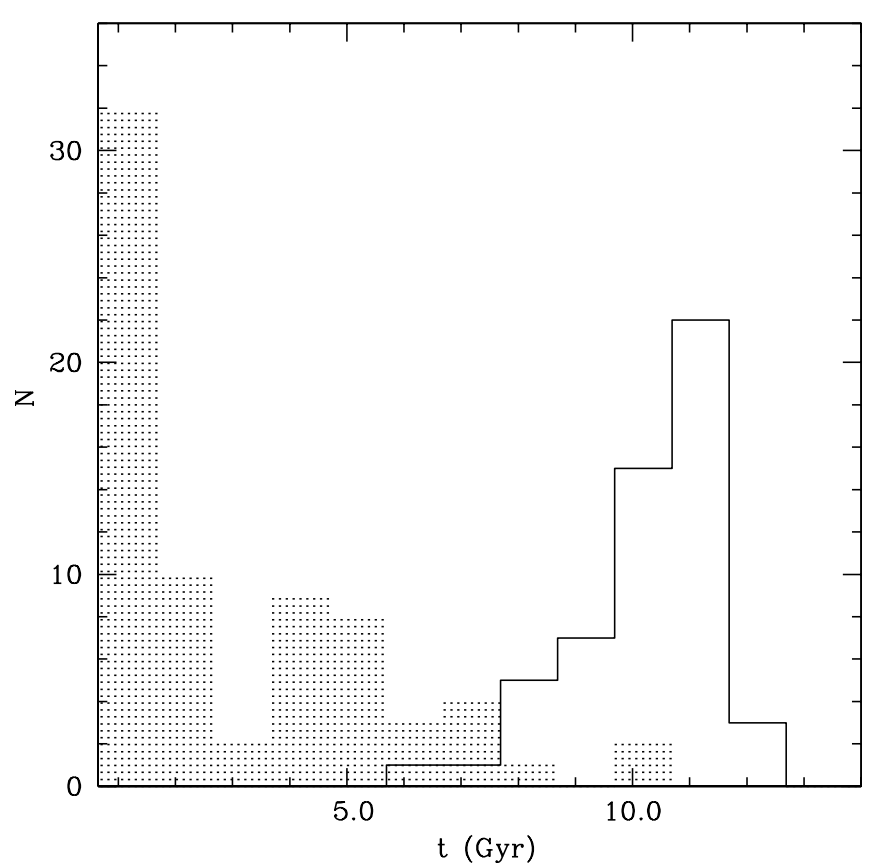

Fig. 11. Histogram of the ages of the GC sample studied by SW02, compared to the OC ages derived in this study (shaded histogram).

of the MS fitting technique using field stars with Hipparcos parallaxes, and the ages obtained from fitting the appropriate isochrone to the absolute brightness of the cluster turn-off region.

To obtain reliable distances and age determinations for the calibrating clusters, a necessary prerequisite is the use of consistent metallicity scales for both field stars and calibrating OCs. The metallicities for the unevolved Hipparcos field dwarfs used in the OC age calibration were derived by PSK03, and shown to be consistent with the G00 metallicity scale for OCs. Comparison between the $[\mathrm{Fe} / \mathrm{H}]$ estimates by $\mathrm{G} 00$ and the recent work by Friel et al. (2002) revealed systematic differences, the most extreme case being NGC 6791, for which the $\mathrm{G} 00$ estimate is $[\mathrm{Fe} / \mathrm{H}]=0.40 \pm 0.06$, whereas Friel et al. found $0.11 \pm 0.10$. A further independent check for the internal consistency of our distances (and ages) is possible, by requiring that the distance to this cluster derived from the MS fitting, is the same when using the $(B-V)$ or the $(V-I)$ colour. The metallicity dependence of the MS colour is different for these two indices (see, e.g. PSK03), therefore the consistency of the distances obtained with $(B-V)$ and $(V-I)$ is a good test for the adopted metallicity scales.

Keeping the cluster $[\mathrm{Fe} / \mathrm{H}]$ as a free parameter, we found that consistent distances are obtained - irrespective of the choice of the cluster reddening - only when $[\mathrm{Fe} / \mathrm{H}]$ is equal to 0.4 , or at least not lower than 0.3 , i.e. when it is homogeneous with the scale used for the dwarfs. With our field dwarf $[\mathrm{Fe} / \mathrm{H}]$ scale a metallicity, e.g., $[\mathrm{Fe} / \mathrm{H}]=0.2$ for NGC 6791 would cause a discrepancy by $0.11 \mathrm{mag}$ between the distances inferred from the $(B-V)$ and $(V-I)$ colours.

With the $\delta(V)-t-[\mathrm{Fe} / \mathrm{H}]$ relation given in Eq. (1), we then derived age estimates for a total of 71 OCs. Their age scale can be merged with the one we published previously for
55 GCs (SW02), 47 Tuc (whose age obtained in this paper agrees with the one estimated using SW02 technique) being the link connecting the two samples. Due to our method, the use of consistent isochrones and an homogeneous metallicity scale, we not only obtained the first large and homogeneous sample of OC ages, but even a reliable age scale on which both cluster types can be placed. This allows the investigation of questions related to the formation of the various components of the Galaxy, halo, thick and thin disk. The bulge still awaits investigation, mainly due to the problem of strong and differential reddening of the bulge cluster CMDs.

Using the whole GC and old OC sample (Fig. 10), we determine a delay by $2.0 \pm 1.5 \mathrm{Gyr}$ between the start of the halo and thin disk formation. We estimated this value by determining the average age (with error) of the two oldest OCs (NGC 6791 and Be 17) - formally coeval - which has been then compared with the age of the oldest metal poor GCs. Liu \& Chaboyer (2000) have estimated 2.8 \pm 1.6 Gyr for this time delay, whereas Carraro et al. (1999) found the thin disk younger than the halo by about 2-3 Gyr, an age difference shorter than the 3-5 Gyr gap determined by Sandage et al. (2003).

We also find that thin and thick disk started to form approximately at the same time, since the age of the thick disk globulars is the same, within errors, as the age of NGC 6791 and $\mathrm{Be} 17$.

The age of the oldest OCs is of the order of $10 \mathrm{Gyr}$, compatible with that of the oldest thin disk white dwarfs as estimated from the white dwarf luminosity function of the solar neighbourhood, which is, according to Hansen (1999), between 6 and 11 Gyr. This rather large age range depends on the uncertainties in the observational data and white dwarf (surface and core) chemical compositions; there are also additional uncertainties due to the white dwarf model physics (e.g. Salaris et al. 2000).

Figure 9 clearly demonstrates the absence of any agemetallicity relation, consistent with earlier results by Carraro \& Chiosi (1994a) and JP94. The overall slope of the relationship between $[\mathrm{Fe} / \mathrm{H}]$ and $R_{\mathrm{gc}}$ is consistent with recent determinations by, e.g., Friel et al. (2002); however, we find a decrease of this slope for increasing cluster ages, which is just the opposite of the results by Friel et al. (2002). We do not detect any correlation between $[\mathrm{Fe} / \mathrm{H}]$ and height above the Galactic plane $|z|$, nor between age and $R_{\mathrm{gc}}$ (as in Carraro \& Chiosi 1994 and JP94).

The cumulative age distribution for the full OC sample shows a departure from the predictions of constant formation rate and exponentially declining dissolution rate (with timescale of $2.5 \mathrm{Gyr}$ ) at the $2 \sigma$ level. No correlation between the cumulative age distribution and $R_{\mathrm{gc}}$ is found; however, there is a significant excess of clusters in the age range between 2-4 and 6 Gyr for the population located at high $|z|$ values, with respect to their counterpart closer to the Galactic plane. It is not clear if this difference is intrinsic - i.e. related to the position of the cluster at its birth - or partly an artifact due to incompleteness of the sample (which, according to JP94, should preferentially affect clusters with lower $|z|$ ) and/or to the cluster orbital motion. 
Acknowledgements. This research has made use of the WEBDA data base (http://obswww. unige.ch/webda). SMP acknowledges financial support from PPARC.

\section{References}

Anthony-Twarog, B. J., \& Twarog, B. A. 1985, ApJ, 291, 595

Carraro, G., \& Chiosi, C. 1994a, A\&A, 287, 761

Carraro, G., \& Chiosi, C. 1994b, A\&A, 288, 751

Carraro, G., Girardi, L., \& Chiosi, C. 1999, MNRAS, 309, 430

Carretta, E., \& Gratton, R. G. 1997, A\&AS, 121, 95

Cassisi, S., Salaris, M., \& Irwin, A. W. 2003, ApJ, 588, 862

Chaboyer, B., Green, E. M., Liebert, J. 1999, AJ, 117, 1360

Edvardsson, B., Andersen, J., Gustafsson, B., et al. 1993, A\&A, 275, 101

Freeman, K., \& Bland-Hawthorn, J. 2002, ARA\&A, 40, 487

Friel, E. D. 1995, ARA\&A, 33, 381

Friel, E. D., Janes, K. A., Tavarez, M., et al. 2002, AJ, 124, 2693

Girardi, L., Bressan, A., Bertelli, G., \& Chiosi, C. 2000, A\&AS, 141, 371

Gratton, R. G. 2000, in Stellar Clusters and Associations: Convection, Rotation, and Dynamos, ed. R. Pallavicini, G. Micela, \& S. Sciortino, ASP Conf. Ser., 198, 225 (G00)

Grevesse, N., \& Noels, A. 1993, in Origin and Evolution of the Elements, ed. N. Prantzos, E. Vangioni-Flam, \& M. Casse (Cambridge: Cambridge University Press), 15

Hansen, B. M. S. 1999, ApJ, 520, 680

Janes, K. A., \& Phelps, R. L. 1994, AJ, 108, 1773 (JP94)

Janes, K. A., Tilley, C., \& Lyngå, G. 1988, AJ, 95, 771

Lejeune, T., \& Schaerer, D. 2001, A\&A, 366, 538
Liu, W. M., \& Chaboyer, B. 2000, ApJ, 544, 818

Mermilliod, J.-C. 1992, Bull. Inf. Centre Donnees Stellaires, 40, 115

Percival, S. M., \& Salaris, M. 2003, MNRAS, 343, 539 (PS03)

Percival, S. M., Salaris, M., van Wyk, F., \& Kilkenny, D. 2002, ApJ, 573, 174 (P02)

Percival, S. M., Salaris, M., \& Kilkenny, D. 2003, A\&A, 400, 541 (PSK03)

Phelps, R. L., \& Schick, M. 2003, AJ, 126, 265

Phelps, R. L., Janes, K. A., \& Montgomery, K. A. 1994, AJ, 107, 1079

Ribas, I., Jordi, C., \& Gimenez, A. 2000, MNRAS, 318 , L55

Rosenberg, A., Saviane, I., Piotto, G., \& Aparicio, A. 1999, AJ, 118, 2306

Salaris, M., \& Weiss, A. 1997, A\&A, 327, 107

Salaris, M., \& Weiss, A. 1998, A\&A, 335, 943

Salaris, M., \& Weiss, A. 2002, A\&A, 388, 492 (SW02)

Salaris, M., Degl'Innocenti, S., \& Weiss, A. 1997, ApJ, 484, 986

Salaris, M., García-Berro, E., Hernanz, M., Isern, J., \& Saumon, D. 2000, ApJ, 544, 1036

Sandage, A., Lubin, L. M., \& Vandeberg, D. A. 2003, PASP, 115, 1187

Sarajedini, A. 1999, AJ, 118, 2321

Sarajedini, A., \& Demarque, P. 1990, ApJ, 365, 219

Spitzer, L. 1958, ApJ, 127, 544

Stetson, P. B., Bruntt, H., \& Grundahl, F. 2003, PASP, 115, 413

Tosi, M. 1996, in From Stars to Galaxies: The Impact of Stellar Physics on Galaxy Evolution, ed. C. Leitherer, U. Fritze-von-Alvensleben, \& J. Huchra, ASP Conf. Ser., 98, 299

Twarog, B. A. 1980, ApJ, 242, 242

Twarog, B. A., Ashman, K. M., \& Anthony-Twarog, B. J. 1997, AJ, 114,2556

Vandenberg, D. A., Bolte, M., \& Stetson, P. B. 1990, AJ, 100, 445 